\title{
Une méthode interactive
}

L'apprentissage du français au Japon

\section{Hajime Sawada}

\section{CpenEdition}

\section{Journals}

Édition électronique

URL : http://journals.openedition.org/ries/2931

DOI : $10.4000 /$ ries. 2931

ISSN : 2261-4265

Éditeur

Centre international d'études pédagogiques

Édition imprimée

Date de publication : 1 juin 1998

Pagination : 101-108

ISSN : 1254-4590

Référence électronique

Hajime Sawada, « Une méthode interactive », Revue internationale d'éducation de Sèvres [En ligne], 18 | juin 1998, mis en ligne le 19 avril 2013, consulté le 06 janvier 2020. URL : http://

journals.openedition.org/ries/2931; DOI : 10.4000/ries.2931

Ce document a été généré automatiquement le 6 janvier 2020.

(c) Tous droits réservés 


\title{
Une méthode interactive
}

\author{
L'apprentissage du français au Japon
}

\author{
Hajime Sawada
}

1 À l'heure où plus d'une cinquantaine d'universités, qu'elles soient nationales ou privées, utilisent des salles multimédias pour l'enseignement des langues étrangères ${ }^{1}$, la question n'est plus de savoir si on doit introduire l'ordinateur dans la classe de langues, mais de chercher à améliorer la pratique pédagogique de CALL (Computer Assisted Language Learning). Ceci est un constat que tout enseignant des langues étrangères au Japon se doit de prendre en compte ${ }^{2}$. Et les professeurs de français, en particulier, ne peuvent ignorer que, chaque année, des dizaines de milliers d'étudiants apprennent l'anglais avec l'aide d'ordinateurs.

2 Pour mieux utiliser les nouvelles technologies et améliorer l'enseignement du français, nous avons adopté, depuis l'ouverture d'un laboratoire de langues multimédias dans notre faculté, la méthodologie New Collaborative Approach. Avant de présenter en détail ce sujet, nous voudrions évoquer certaines raisons pour lesquelles CALL doit être nécessairement exploité dans un établissement ayant en vue la formation des citoyens du XXI ${ }^{\mathrm{e}}$ siècle. Ces raisons sont d'ordre social et pédagogique.

\section{Plurilinguisme et savoir dans la société de l'information}

3 Les industries dans le domaine des nouvelles technologies de l'information et de la communication sont en plein essor dans beaucoup de pays du monde. On constate un peu partout une sorte de boom du multimédia et de l'Internet. Les enjeux de ce développement ne sont pas seulement économiques. Selon certains commentateurs, la vulgarisation au niveau grand public de ces nouvelles technologies annonce l'avènement d'une société électronique informatisée et ouvre les perspectives d'une révolution cognitive aussi importante que la découverte de l'imprimerie par Gutenberg. 
4 Aujourd'hui, la langue commune sur Internet est l'anglais, domination linguistique que l'on peut considérer comme naturelle dans un premier temps, Internet étant un produit de la culture américaine. Mais notre tâche est d'établir le plurilinguisme sur ce formidable réseau de l'information et de la communication. Pour que le plurilinguisme nécessaire règne, il faut que le français soit reconnu en tant que langue internationale sur le réseau des réseaux. Cela ne signifie ni que l'on doive ni que l'on puisse négliger les autres langues. En dehors de l'anglais et loin derrière lui, le français reste la seule langue de communication à l'échelle mondiale. La France est un des rares pays avancés qui soit conscient de la nécessité de préserver avec volonté les langues et les cultures existant de par le monde. Si le français, langue du pays promoteur du plurilinguisme, ne devenait pas une deuxième langue commune sur Internet, nous ne pourrions jamais espérer la diversification de l'information et de la communication dans l'ère de la culture technologique informatisée.

5 La familiarisation aux nouvelles technologies et l'acquisition des langues étrangères sont donc indispensables aux jeunes du XXI ${ }^{e}$ siècle. Les professeurs de langues peuvent contribuer à la formation de la jeunesse, quand ils sont conscients que le multimédia n'est pas seulement un nouveau gadget audiovisuel, mais un système qui intègre les divers moyens de transmission de l'information et les divers supports d'expression. Ils peuvent assumer leur rôle par leurs pratiques de CALL. Et les professeurs de français, en encourageant les apprenants à conduire des activités créatrices en milieu multimédia, mettent du même coup en valeur l'usage du français dans la construction de nouvelles cultures.

6 Ainsi, nous devons résolument prendre le parti d'utiliser l'ordinateur pour l'enseignement/apprentissage du français à l'ère de la culture technologique informatisée. C'est dans cette perspective que nous avons poursuivi l'étude des possibilités actuelles de l'enseignement multimédia du français et que nous avons introduit au sein de notre enseignement des activités multiples qui s'appuient sur les apports de l'ordinateur.

\section{NEW COLLABORATIVE APPROACH AU SERVICE DU FLE}

7 Notre faculté s'est dotée en 1993 d'une salle multimédia, spécialement conçue pour l'enseignement des langues étrangères. Son système intègre les fonctions du laboratoire de langues et les fonctions d'un réseau de communication câblé connecté sur Internet. Nous disposons aussi depuis 1997 d'un centre de ressources multimédias pour l'apprentissage des langues étrangères. Outre un libre accès à Internet, les étudiants peuvent consulter différents didacticiels et produire eux-mêmes des documents multimédias ${ }^{3}$.

8 Pour illustrer l'intégration de l'ordinateur dans notre classe de français, nous présenterons d'abord notre approche pédagogique et donnerons ensuite quelques applications.

\section{Approche complexe}

9 Nous intervenons dans une classe commune de français qui se tient quatre fois par semaine, pendant deux ans. Dès le début de CALL dans notre établissement, nous avons adopté comme méthode principale une méthode audiovisuelle pour cette classe. Cette 
méthode, grâce à la qualité du film feuilleton, suscite spontanément un intérêt très vif et une motivation forte chez les débutants complets, ce qui est difficilement réalisable même avec les manuels plus récents et plus novateurs. Elle présente, en outre, une abondance d'éléments à apprendre sur les plans référentiel, culturel et linguistique. Mais nous n'avançons pas notre programme en suivant mot à mot le guide pédagogique. Nous ne traitons pas toutes les questions consignées dans le cahier d'exercices. Nous donnons aux étudiants des manuels auxiliaires, des polycopiés ou des fiches par réseau concernant la grammaire, la prononciation, le vocabulaire ou la lecture. La méthode nous sert à la fois de fil conducteur pour l'ensemble des éléments didactiques et de déclencheur, selon des modalités diverses, en vue d'une exploitation supplémentaire ou approfondie.

Différentes méthodologies alternent au cours d'une leçon selon ce que nous voulons enseigner ou animer. Un découpage des activités pédagogiques distinguerait différents moments: méthode grammaire-traduction, méthode directe, méthode audio-orale, approche notionnelle-fonctionnelle ou approche communicative. La grammaire, la lecture, l'écriture, la conversation ou l'écoute, rien n'est négligé dans la classe et chaque aspect est plus abondamment exploité selon les cours ou les périodes donnés.

11 Notre méthodologie peut relever de l'éclectisme. Dans cette pratique pédagogique où l'on conjugue des efforts pour construire une classe adaptée aux apprenants selon la situation d'une année donnée, l'outil indispensable pour nous est l'ordinateur. Christian Puren prône une "didactique complexe» qui, dans le processus d'enseignement/apprentissage d'une langue/culture, permet aux enseignants de " construire eux-mêmes et pour eux-mêmes (et pour les apprenants...) de multiples cohérences partielles, provisoires et locales et de les articuler les unes aux autres $»^{4}$. Nous considérons que CALL permet de concrétiser de la manière la plus riche cette approche complexe dans les séquences pédagogiques.

Le système multimédia assisté par ordinateur peut assurer séparément les fonctions offertes par une salle audiovisuelle, une salle d'ordinateurs et un laboratoire de langues. Mais le multimédia n'est pas une simple addition de médias. En intégrant les fonctions de ces trois modalités d'apprentissage, le laboratoire de langues multimédias permet aussi, en une même heure, de passer librement d'un type d'activité à l'autre selon le contenu de la leçon. De plus, il rend possible la gestion simultanée de différents types d'apprentissage; au cours d'une même période, les étudiants peuvent choisir leur activité selon leurs besoins. Il permet de mettre en place un enseignement plus interactif jusqu'alors difficilement réalisable.

13 CALL est donc à même de réaliser :

- une classe adaptée au niveau de connaissances de chaque apprenant ;

- une classe pour dispenser un entraînement efficace à l'ensemble des apprenants ;

- une classe riche en interactivités ;

- une classe ouverte à la créativité.

Ce constat nous conduit à penser qu'une des méthodologies les plus prometteuses pour l'avenir du FLE est l'enseignement/apprentissage d'une langue en collaboration entre enseignant, apprenant et ordinateur. Un travail d'équipe qui associe professeurs français et professeurs japonais, conjugué à des travaux de groupes en vue de la production de réalisations collectives par les étudiants assistés par un réseau 
d'ordinateurs et des programmes susceptibles de répondre en temps utile aux besoins linguistiques de chacun, voilà ce que nous entendons par New Collaborative Approach.

Cette approche a pour base l'idée de la centration sur l'apprenant. Celle-ci existait déjà dans les méthodologies qu'on élaborait dans les années soixante-dix. Mais elle prend une nouvelle signification dans le contexte actuel de transformation de la société et de changement de la pensée pédagogique. Les apprenants étudient une langue étrangère dans un environnement multimédia et y cherchent des informations en utilisant cette langue pour arriver à rendre public le résultat de leurs activités et à communiquer avec les autres. Ceci s'accompagne d'interactions plus ou moins importantes entre les apprenants à chaque étape. La classe de langues est ainsi appelée, elle aussi, à faire partie d'un espace ouvert sur le monde.

À l'université nationale de Hiroshima, nous avons essayé de construire ce système de l'enseignement/apprentissage d'une langue/culture pour une classe de français. Les quatre cours par semaine sont pris en charge par quatre professeurs, deux Français et deux Japonais. Dans notre équipe, un professeur japonais et un professeur français utilisent le laboratoire de langues multimédias. Mais, si elle reste cantonnée à l'intérieur de la salle de classe, l'utilisation de l'ordinateur est problématique et ne satisfait pas pleinement aux besoins des apprenants. C'est pourquoi nous donnons des suggestions et des propositions pour les activités autonomes qu'ils peuvent accomplir au centre de ressources multimédias pour l'apprentissage des langues étrangères. Ces suggestions et propositions sont conçues en sorte qu'ils puissent faire le travail sous forme de découverte, recherche, expression ou création. À part le libre choix laissé aux étudiants, nous leur donnons intentionnellement des tâches telles que la réalisation d'enregistrements de dialogues écrits par eux-mêmes ou la fabrication autonome de programmes pour la lecture de textes en français. Car nous pensons que les apprenants ne doivent pas rester de simples récepteurs, mais qu'ils doivent devenir créateurs de produits multimédias. C'est à l'école qu'ils doivent acquérir cette aptitude. Cependant, les exemples de notre application pédagogique de CALL comportent des activités qui reposent sur des méthodes traditionnelles. Instruire et entraîner restent nécessaires dans certaines séquences pédagogiques.

\section{Applications pédagogiques de CALL 5}

\section{Une classe adaptée au niveau de connaissances de chaque apprenant}

Pour familiariser les étudiants avec l'emploi des logiciels éducatifs dont les modes d'exercices ne sont pas aussi faciles à comprendre qu'on le supposerait, nous utilisons dans la classe, au début du premier semestre, des cédéroms pour l'apprentissage du vocabulaire, qui intègrent des graphismes, des textes et du son.

Les étudiants apprennent le vocabulaire, à leur rythme, à travers divers types d'exercices. De notre côté, avec des fonctions traditionnelles du laboratoire de langues, nous dialoguons individuellement avec eux et corrigeons leur prononciation. Au terme de cette période d'initiation, les étudiants peuvent choisir des didacticiels au centre de ressources multimédias. 


\section{Une classe pour dispenser un entraînement efficace à l'ensemble des apprenants}

19 Une bonne exploitation de la méthode utilisée suppose l'ajout d'exercices qui gagnent souvent à être conçus sur ordinateur. Nous avons fabriqué ainsi plusieurs didacticiels, dont la terminologie "Nom des pays d'Europe ». Celle-ci est constituée d'une dizaine de cartes. Les étudiants peuvent apprendre, à travers divers types d'exercices, l'endroit géographique, l'orthographe, la prononciation des pays d'Europe.

Le but de ces petits programmes est de faire apprendre d'une manière intensive le vocabulaire relevant de thèmes précis pour qu'ils puissent le réutiliser effectivement dans les jeux de rôles ou d'autres activités de la classe.

\section{Une classe riche en interactivité}

21 Au cours du premier mois du semestre, nous demandons aux étudiants d'envoyer leur fichier de présentation vers le serveur. Nous mettons le texte original d'un étudiant sur la partie gauche de l'écran et le double de ce texte sur la partie droite pour transmettre l'image sur tous les terminaux. Les passages qui contiennent des fautes sont soulignés, tandis que nous commentons tout en corrigeant le double du texte. Les étudiants observent ce déroulement sur leur écran et répondent à des questions concernant ces passages.

$22 \mathrm{Au}$ deuxième mois du semestre, nous leur donnons comme tâche de travailler en groupe, pour écrire la suite d'un scénario très court. Après un travail de compréhension orale et écrite des scénarios achevés par les groupes, ceux-ci doivent, à la fin du semestre, jouer leur sketch devant la classe et sous l'œil du caméscope. L'objectif principal de ces démarches est de familiariser les étudiants avec le travail en commun ainsi que de développer leur capacité d'expression.

\section{Une classe créative}

23 Au cours de leur deuxième année d'études, nous demandons que les étudiants utilisent Internet pour créer des documents interactifs. Nous leur demandons d'accomplir une tâche telle que la présentation d'un projet de voyage dans une province en France à l'aide des équipements du laboratoire de langues multimédias.

24 Nous avons aussi, en dehors des cours de français normaux, plusieurs travaux pratiques. Dans cette classe, le travail individuel va de pair avec le travail collectif.

25 L'apprentissage individuel passe par l'utilisation d'un cédérom. Outre l'intérêt des histoires interactives, ce logiciel de lecture offre aux lecteurs des séries d'exercices qui permettent la prise de conscience systématique de différents aspects du fonctionnement de la langue française.

26 L'apprentissage collectif consiste à créer en groupe un conte en français. Depuis que nous avons lancé cette expérience en 1995, nous avons pu observer que la création du conte en milieu multimédia et en groupe a toujours ouvert un espace de communication très active entre les apprenants. Ceux-ci acquièrent des compétences en lecture et en expression à travers la discussion et le travail en commun que nécessite la mise en forme d'un conte original.

Les exemples donnés ci-dessus ne présentent que quelques aspects des activités conduites dans notre cours. Si nous ne mentionnons pas les activités qu'on peut animer 
en utilisant le courrier électronique à partir des éléments très riches qui se trouvent dans la méthode utilisée, c'est qu'un de nos collègues français se charge de ce travail. Il anime par ailleurs un cours intitulé Travaux pratiques de conversation en français pour le niveau avancé qui propose une exploitation plus systématique des ressources offertes par Internet. Nous accomplissons chacun nos tâches qui se complètent dans l'ensemble des programmes d'études. meilleur. Nous admettons volontiers que l'anglais est une langue commune sur le cyberespace. Mais nous pensons que d'autres langues doivent y être présentes d'une manière bien visible. Nous devons plus et mieux utiliser l'ordinateur dans le cadre de l'enseignement d'une deuxième langue étrangère.

31 En guise d'ultime conclusion, nous voudrions finir notre article en formulant un souhait vers la France. Nous aimerions que tous ceux qui sont concernés par la diffusion de la langue et de la culture françaises fassent tout leur possible pour rendre la France de demain plus rayonnante que jamais. Et cela dans le cyberespace également. Nous désirerions voir la France devenir un grand centre d'émission d'informations sur Internet. Elle doit encourager la création de sites riches en informations de toutes sortes, sous forme non seulement de textes et de graphiques, mais aussi de programmes radio et d'émissions télévisées gratuites, comme on en voit tant sur les réseaux américains. Car, désormais, on ne saurait motiver longtemps les gens à étudier une langue si celle-ci ne diffuse pas constamment des informations originales et stimulantes. 


\section{NOTES}

1. Sans compter des écoles des langues privées qui investissent dans des équipements multimédias et dont certaines vont jusqu'à produire des cédéroms éducatifs.

2. Pour plus de détails en ce qui concerne l'état présent de la pratique de CALL au Japon, voir notre article : « Multimédia et évolution de l'enseignement des langues étrangères au Japon », in The Dong-eui International Journal 2, Institute of Foreign Language Research and Education, Dongeui University, Corée, 1996, p. 121-143.

3. Au début de leur première année universitaire, les étudiants reçoivent automatiquement une adresse électronique et un mot de passe. Tant qu'ils utilisent l'ordinateur à l'intérieur de l'université, ils peuvent bénéficier gratuitement des services d'Internet et du courriel.

4. Christian Puren, "Éclectisme et complexité », Cahiers pédagogiques. «Le FLE, une langue vivante ", n³60, 1998, p. 15.

5. Pour le détail de certaines pratiques, se reporter à l'article cité plus haut ainsi qu'à notre article : «Multimédia et ordinateurs : les perspectives dans l'enseignement futur du français », Dialogues et cultures 41, Actes du IX Congrès mondial de la Fédération internationale des professeurs de français, 1997, p. 462-468.

\section{RÉSUMÉS}

L'emploi des nouvelles technologies est aujourd'hui incontournable, notamment dans l'enseignement des langues. La New Collaborative Approach est une méthodologie mise en place à l'université nationale d'Hiroshima pour l'enseignement de la langue française. Riche en créativité et en interactivités, la classe peut ainsi être adaptée au niveau de chaque étudiant.

\section{INDEX}

Index géographique : Japon

Mots-clés : nouvelles technologies, usage didactique de l'ordinateur, multimédia, pédagogie, apprentissage du FLE, TICE : technologies de l'information et de la communication en éducation

\section{AUTEUR}

\section{HAJIME SAWADA}

Maître de conférences, section de français, université nationale d'Hiroshima, Japon 